\title{
Clinical significance of serum laminin levels in patients with lung cancer
}

\author{
FARUK TAS, ELIF BILGIN, DIDEM TASTEKIN, KAYHAN ERTURK and DERYA DURANYILDIZ \\ Department of Medical Oncology, Institute of Oncology, Istanbul University, Capa, Istanbul 34390, Turkey
}

Received December 7, 2015; Accepted February 17, 2016

DOI: $10.3892 /$ br.2016.612

\begin{abstract}
Cellular adhesion molecules are considered useful markers in the diagnosis and prognosis of several types of malignant tumors. Laminin, a major structural component of the basement membrane, is a strong promoter of cell adhesion, migration, differentiation and proliferation via integrins and other cell surface receptors. The present study aimed to evaluate the clinical significance of the serum level of laminin in lung cancer patients. A total of 80 patients with lung cancer were enrolled in the study. The serum laminin level was measured by the solid-phase sandwich enzyme-linked immunosorbent assay method. The median age was 58.5 years (range, 36-80 years). The majority of the patients had non-small cell lung carcinoma $(85 \%)$ and stage IV disease $(56 \%)$. The baseline serum laminin levels of patients were significantly higher compared to the control group (median values 1.17 vs. $0.78 \mathrm{ng} / \mathrm{ml}, \mathrm{P}=0.033$ ). However, the clinical variables, such as age, gender, histology, stage of disease and response to chemotherapy, were not correlated with serum laminin level $(\mathrm{P}>0.05)$. Similarly, serum laminin level was not associated with survival $(\mathrm{P}=0.68)$. In conclusion, the serum level of laminin may have a diagnostic value in lung cancer patients. However, its predictive and prognostic roles were not observed.
\end{abstract}

\section{Introduction}

Lung cancer is a highly invasive malignancy that has a strong tendency to metastasize at early stages. Therefore, the interaction of the lung cancer cells with the extracellular matrix (ECM) in the way of cell adhesion, migration, proliferation and metastasis has been observed. Laminin, one of the major structural components of the basement membrane, is a strong promoter of cell adhesion, migration, differentiation and proliferation by means of integrins and other cell

Correspondence to: Dr Faruk Tas, Department of Medical Oncology, Institute of Oncology, Istanbul University, 118 Millet Street, Capa, Istanbul 34390, Turkey

E-mail: faruktas2002@yahoo.com

Key words: laminin, serum, lung cancer, diagnostics surface receptors (1-4). Increased laminin expression was also detected in lung cancer cell lines with increased lung cancer cell proliferation and metastatic potential (1-4).

Although there are increasing data regarding the role of laminin in various malignancies, only a few studies of laminin expression in lung cancer have been reported (1-3). However, the current available findings have been provided from preclinical tissue or cell-based trials. As no clinical study has investigated the laminin level in serum and/or plasma in lung cancer patients, the significance of the serological level of laminin in this group of patients remains to be elucidated.

Therefore, the soluble serum laminin levels in patients with lung cancer, were evaluated, and their association with prognosis, various known clinical variables and response to chemotherapy were assessed, so as to elucidate whether this biomarker may be useful in making the diagnosis and in the assessment of the prognosis.

\section{Materials and methods}

Patients. A total number of 80 patients with histologically or cytologically confirmed non-small cell lung cancer (NSCLC) and SCLC treated and followed up in the Institute of Oncology (Istanbul University, Istanbul, Turkey) were enrolled in the study. The patients had bidimensionally measurable disease without a history of chemo/radiotherapy in the last 6 months. The metastatic diseases were staged with various imaging modalities such as computed tomography, magnetic resonance imaging and positron emission tomography/computed tomography scan. The pathological diagnosis of lung cancer was established according to the revised World Health Organization classification of lung tumors $(5,6)$ and staged relying on the revised tumor-node-metastasis staging for lung cancer.

The clinical history, physical examination, series of biochemistry tests and complete blood cell counts were used as the pretreatment evaluation. Those with Eastern Cooperative Oncology Group performance status $\leq 2$ and appropriate blood chemistry tests received a platinum-based chemotherapy with/without radiotherapy depending on the stage of disease. The response to chemotherapy was evaluated radiologically after 2-3 cycles of chemotherapy according to revised Response Evaluation Criteria in Solid Tumors criteria (7). The non-responders to chemotherapy and patients with recurrent diseases were treated with second-line chemotherapy provided 
when they had a good performance status. Chemotherapy was discontinued when disease progression or unacceptable toxicity occurred.

A total of 30 healthy age- and gender-matched controls were included in the analysis. The study was approved by the ethics committee of the Institute of Oncology. Written informed consent was obtained from all the patients.

Measurement of serum laminin levels. Serum samples were drawn from patients and healthy controls by venipuncture and clotted at room temperature on first admission prior to the treatment. The sera were collected following centrifugation at room temperature for $10 \mathrm{~min}$ at $4,000 \mathrm{rpm}$ and frozen immediately at $-20^{\circ} \mathrm{C}$ until analysis.

Serum laminin levels were measured by the solid-phase sandwich enzyme-linked immunosorbent assay (ELISA) method that used double-antibody sandwich ELISA. Serum samples and standards were added to the wells that were pre-coated with human laminin monoclonal antibody (cat. no. E0082h; Wuhan EIAab Science Co., Ltd., Wuhan, China). Following incubation, laminin antibodies labeled with biotin and combined with streptavidin-horseradish peroxidase were added to form an immune complex and incubated for $1 \mathrm{~h}$. Unbound material was washed away and subsequently the chromogen solution was added for the conversion of the colorless solution to a blue solution, the intensity of which was proportional to the amount of laminin in the sample. The color changed to yellow as a result of the acidic stop solution. The colored reaction product was measured using an automated ELISA reader (ChroMate, 4300 Microplate Reader; Awareness Technology, Inc., Palm City, FL, USA). The results are expressed as $\mathrm{ng} / \mathrm{ml}$.

Statistical analysis. Median values were used to classify the variables and the Mann-Whitney U test was used to compare the clinical and laboratory parameters. Survival was calculated from the first admission date to the date of fatality from any cause or to the last contact with the patient or any family member. Kaplan-Meier test was used to estimate the survival and the differences in survival were evaluated by the log-rank statistics. $\mathrm{P} \leq 0.05$ was considered to indicate a statistically significant difference. Statistical analysis was carried out using SPSS 16.0 software (SPSS, Inc., Chicago, IL, USA).

\section{Results}

Patient characteristics. A total of 80 consecutive patients with pathologically confirmed diagnosis of lung cancer were enrolled in the study. Baseline histopathological and demographic data of patients are listed in Table I. The median age of patients was 58.5 years (range, 36-80 years), and males constituted the majority of the group $(\mathrm{n}=72,90 \%)$. The majority of the patients had NSCLC $(\mathrm{n}=68,85 \%)$ and metastatic disease $(\mathrm{n}=45,56 \%)$.

Serum laminin. The levels of serum laminin in patients and healthy controls are shown in Table II. The baseline serum laminin concentrations of the lung cancer patients were significantly higher than those in the control group (median values 1.17 vs. $0.78 \mathrm{ng} / \mathrm{ml}, \mathrm{P}=0.033$ ) (Fig. 1).
Table I. Patient characteristics and disease status.

\begin{tabular}{|c|c|}
\hline Variables & Total, \\
\hline No. of patients & 80 \\
\hline \multicolumn{2}{|c|}{ Age of patients, years } \\
\hline$\geq 60$ & 37 \\
\hline$<60$ & 43 \\
\hline \multicolumn{2}{|l|}{ Gender } \\
\hline Male & 72 \\
\hline Female & 8 \\
\hline \multicolumn{2}{|l|}{ Histology } \\
\hline NSCLC & 68 \\
\hline Adenocarcinoma & 33 \\
\hline Squamous cell & 27 \\
\hline Undifferentiated & 8 \\
\hline SCLC & 12 \\
\hline \multicolumn{2}{|l|}{ Stage } \\
\hline II & 4 \\
\hline III & 30 \\
\hline IV & 34 \\
\hline Limited & 1 \\
\hline Extended & 11 \\
\hline \multicolumn{2}{|c|}{ Response to chemotherapy } \\
\hline Yes & 41 \\
\hline No & 30 \\
\hline
\end{tabular}

NSCLC, non-small cell lung carcinoma.

Table II. Values of serum laminin levels in the lung cancer patients and healthy controls.

\begin{tabular}{llll}
\hline & \multicolumn{2}{c}{ Median laminin (range), ng/ml } & \\
\cline { 2 - 3 } Assay & Patients, $\mathrm{n}=80$ & Controls, $\mathrm{n}=30$ & P-value \\
\hline $\begin{array}{l}\text { Serum laminin } \\
\text { level }\end{array}$ & $1.17(0.12-310)$ & $0.78(0.22-1.56)$ & 0.033 \\
\hline
\end{tabular}

Correlation between serum laminin levels and the clinicopathological variables. Table III shows the correlation between the serum laminin levels and clinicopathological variables. Known clinical variables including age of patient, gender, histology, stage of disease and response to chemotherapy were not correlated with serum laminin concentrations $(\mathrm{P}>0.05)$.

Survival. The median follow-up time was 58 weeks (range, 3.7-149.3 weeks). The median survival was 94.4 weeks (95\% confidence interval: 73.1-115.7). The 1- and 2-year overall survival rates were 68.5 and $40.6 \%$, respectively. Histology $(\mathrm{P}=0.004)$, metastasis $(\mathrm{P}=0.005)$ and response to chemotherapy $(\mathrm{P}=0.009)$ had prognostic factors on 
Table III. Distribution and survival comparisons of the serum laminin levels on various clinical parameters in patients with lung cancer.

\begin{tabular}{lcc}
\hline & \multicolumn{2}{c}{$\begin{array}{c}\text { Serum laminin } \\
\text { level, P-value }\end{array}$} \\
\cline { 2 - 3 } Parameters & Distribution & Survival \\
\hline $\begin{array}{l}\text { Age of patients, years } \\
\geq 60 /<60\end{array}$ & 0.16 & 0.43 \\
$\begin{array}{l}\text { Gender } \\
\text { Male/female }\end{array}$ & 0.11 & 0.75 \\
$\begin{array}{l}\text { Histology } \\
\text { NSCLC/SCLC }\end{array}$ & 0.83 & 0.004 \\
Histology in NSCLC & & 0.81 \\
$\begin{array}{l}\text { Adeno/epidermoid } \\
\text { Clinical stage } \\
\text { Non-metastatic (I-III)/ } \\
\text { metastatic (IV) }\end{array}$ & 0.24 & 0.005 \\
$\begin{array}{l}\text { Response to chemotherapy } \\
\text { Yes/no }\end{array}$ & 0.24 & \\
$\begin{array}{l}\text { Serum laminin level } \\
\text { Median, < or } \geq\end{array}$ & 0.48 & 0.009 \\
\hline
\end{tabular}

NSCLC, non-small cell lung carcinoma.

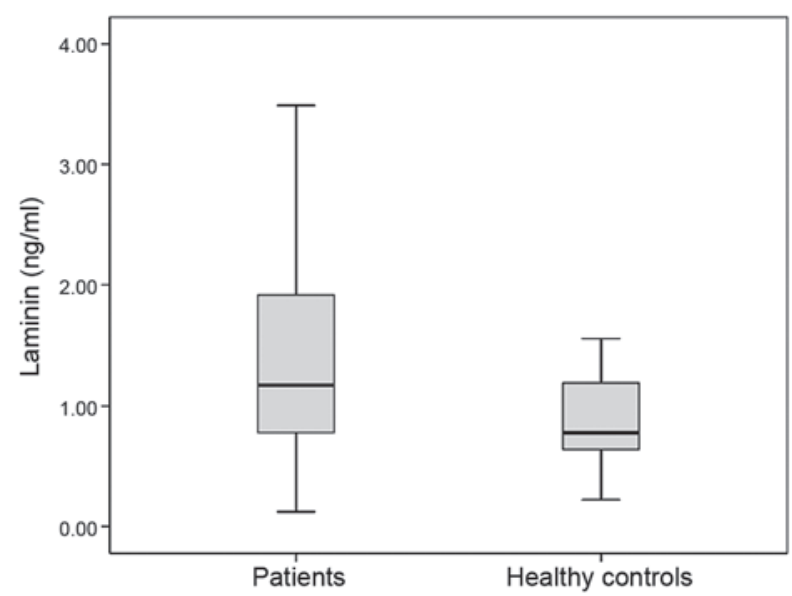

Figure 1. Values of the serum laminin assay in the lung cancer patients and healthy controls $(\mathrm{P}=0.033)$.

survival (Table III). However, the serum laminin level was not associated with survival ( $\mathrm{P}=0.68)$ (Table III, Fig. 2).

\section{Discussion}

There are limited studies on the role of laminin in lung cancer that are limited to paraffin-embedded materials and cell line trials; therefore, all the available data have been provided from tissue cultures. However, the present study was carried out in serum instead of tissue, which to the best of our knowledge is a first.

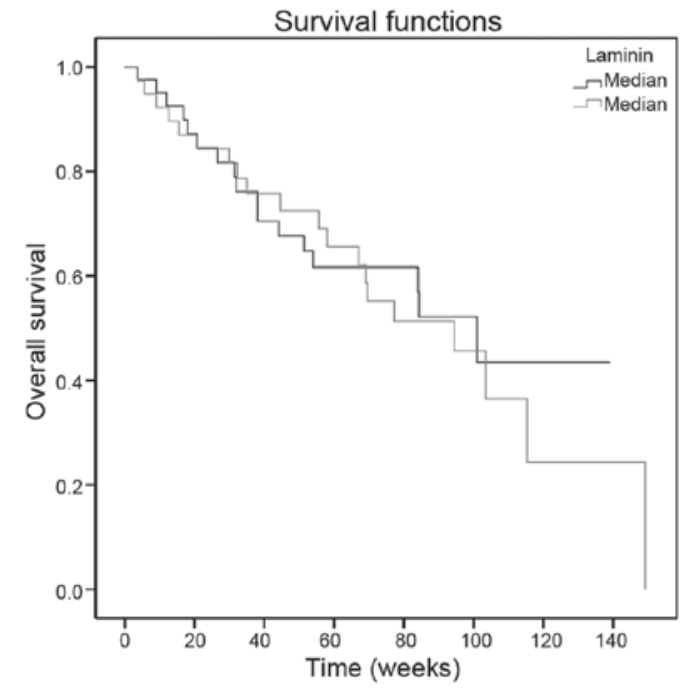

Figure 2. Survival curves in the lung cancer patients according to the serum laminin levels $(\mathrm{P}=0.68)$.

Using monoclonal antibodies, laminin expression as a basement membrane component and keratin intermediate filament protein in normal human bronchial epithelium and 56 lung carcinomas was assessed by Wetzels et al (1). In normal lung tissues, laminin was located around the alveoli and beneath the epithelial basal cell layer in bronchi and bronchioles. Laminin was stained in all the lung cancer subtypes, such as squamous cell carcinoma, adenocarcinoma, small cell lung carcinomas and carcinoids.

$\mathrm{Xu}$ et al (2) studied the expression of ECM proteins in 57 formalin-fixed, paraffin-embedded human NSCLC specimens and compared them with 23 normal lung tissues. The positive expression rate of laminin was higher in NSCLC stroma compared to that in normal lung tissue, 54.4 and $26.1 \%$, respectively $(\mathrm{P}<0.05)$. In $19.3 \%$ of the tumors, laminin was stained intracellularly as well. The expression rates of laminin in well- and moderately differentiated NSCLC were higher compared to those in poorly differentiated cancers $(\mathrm{P}<0.05)$. Additionally, laminin was more frequently observed in squamous cell carcinoma compared to adenocarcinoma $(\mathrm{P}<0.05)$ and a higher expression rate of laminin was associated with node-negative disease $(\mathrm{P}<0.05)$. However, there was no correlation between the expression of laminin and the stage of disease.

Szelachowska et al (3) analyzed the hypothesis that the level of intracellular laminin was of prognostic importance in NSCLC by designing an immunohistochemical (IHC) study. The increased level of intracellular laminin and the presence of laminin in $>50 \%$ of cells caused a significantly reduced patient survival. Thus, the increased laminin accumulation is associated with the progression of the malignancy.

These conflicting results may be attributable to several factors in that, for example, no consensus exists on which tumors and methods should be used to test the expression of laminin. Recently, IHC has been increasingly used as an adjunctive method in diagnostic histopathology. However, there are limitations with IHC, the most important of which are a lack of assay standardization and variance in the interpretation of the IHC staining. In a number of cases, the studies were 
performed on a relatively small sample size, which may have been insufficient to illustrate significant differences.

Proteases degrade laminin into several fragments. Laminin P1 is one such fragments that is chemically a pepsin-resistant soluble peptide (4). A sensitive radioimmunoassay for the laminin P1 fragment showed that laminin had cross-reactivity with its fragment P1. Nakano et al (4) measured the serum laminin P1 level in 43 lung cancer patients, in individuals with benign lung disease and in normal subjects with a radioimmunoassay that was sensitive for the laminin P1 fragment, and detected the cross-reactivity between laminin and its fragment (4). The level of serum laminin P1 was elevated in 58.9 and $11.5 \%$ of SCLC and NSCLC patients, respectively. The values of laminin P1 in SCLC patients were significantly higher than those in the NSCLC patients $(\mathrm{P}<0.01)$ and in the patients with respiratory infection $(\mathrm{P}<0.01)$, and also those in healthy individuals $(\mathrm{P}<0.01)$. Similarly, serum laminin $\mathrm{P} 1$ in SCLC was correlated with the chemotherapeutic response. However, no correlation was identified between the laminin P1 level and clinical stage of SCLC.

A total of 80 patients with different histology and stages of lung cancer were enrolled in the present study. The serum laminin levels of the lung cancer patients were significantly higher than those in the control group. However, clinical variables, such as age, gender, site of lesion, histology, stage, serum $\mathrm{LDH}$ levels and response to chemotherapy, were not correlated with the serum laminin concentrations. Additionally, laminin did not have a prognostic role in survival in lung cancer.

In conclusion, the present study concurs with the previous studies that have investigated laminin in tissue, and serum laminin levels may have a diagnostic role in lung cancer. Additionally, laminin had no predictive and prognostic values. Although the small sample size and the short follow-up time are limitations and may have influenced the results, this study contributes significant information in that it was carried out with serum instead of tissue and it evaluated all stages of the disease. Larger scale studies in larger patient populations are required to determine the exact role of serum laminin in lung cancer patients.

\section{References}

1. Wetzels RHW, Schaafsma HE, Leigh IM, Lane EB, Troyanovsky SM, Wagenaar SSC, Vooijs GP and Ramaekers FCS: Laminin and type VII collagen distribution in different types of human lung carcinoma: Correlation with expression of keratins 14, 16, 17 and 18. Histopathology 20: 295-303, 1992.

2. Xu Y, Zhao Y, Su B, Chen Y and Zhou C: Expression of collagen IV, fibronectin, laminin in non-small cell lung cancer and its correlation with chemosensitivities and apoptosis. Chinese-German J Clin Oncol 5: 58-62, 2006.

3. Szelachowska Y, Jelen M and Kornafel J: Prognostic significance of intracellular laminin and Her $2 /$ neu overexpression in non-small cell lung cancer. Anticancer Res 26: 3871-3876, 2006.

4. Nakano T, Iwahashi N, Maeda J, Hada T and Higashino K: Serum laminin P1 in small cell lung cancer: a valuable indicator of distant metastasis? Br J Cancer 65: 608-612, 1992.

5. Brambilla E, Travis WD, Colby TV, Corrin B and Shimosato Y: The new World Health Organization classification of lung tumours. Eur Respir J 18: 1059-1068, 2001.

6. Detterbeck FC, Boffa DJ and Tanoue LT: The new lung cancer staging system. Chest 136: 260-271, 2009.

7. Eisenhauer EA, Therasse P, Bogaerts J, Schwartz LH, Sargent D, Ford R, Dancey J, Arbuck S, Gwyther S, Mooney M, Rubinstein L, Shankar L, Dodd L, Kaplan R, Lacombe D and Verweij J: New response evaluation criteria in solid tumours: revised RECIST guideline (version 1.1). Eur J Cancer 45: 228-247, 2009. 